\title{
Variante plasmocitoide del carcinoma urotelial: a propósito de un caso
}

\section{Plasmacytoid variant of urothelial carcinoma: a case report}

\author{
J.M. Velis' ${ }^{1}$, J.F. Barba ${ }^{1}$, A. Tienza ${ }^{1}$, F.J. Queipo ${ }^{2}$, J.J. Sola ${ }^{2}$, J.J. Zudaire ${ }^{1}$
}

\section{RESUMEN}

El carcinoma urotelial plasmocitoide es un hallazgo anatomopatológico extremadamente raro. Aportamos nuestra experiencia de un caso.

Varón de 60 años con episodios de hematuria de 2 años de evolución con polaquiuria y disuria. Se evidenció un tumor realizando primero una RTU vesical cuyo diagnóstico anatomopatológico fue de carcinoma urotelial plasmocitoide, por lo que posteriormente se realizó una cisto-prostatectomía radical con derivación ileal, y tras más de dos años de evolución falleció.

\begin{abstract}
Plasmacytoid urothelial carcinoma is an extremely rare pathological finding. We report our experience of one case.

A 60 year old male with hematuria of two years evolution, with frequency and dysuria. A tumor was found and he received surgical treatment by TURB at first. The pathology result was a plasmacytoid urothelial carcinoma. Subsequently a radical cystectomy with urinary diversion was performed. The patient received follow-up until his death.
\end{abstract}

1. Departamento de Urología. Clínica Universidad de Navarra

2. Departamento de Anatomía Patológica. Clínica Universidad de Navarra

Recepción: 5 de diciembre de 2012

Aceptación provisional: 15 de enero de 2013

Aceptación definitiva: 1 de febrero de 2013
Correspondencia:

Departamento de Urología

Clínica Universidad de Navarra

31008 Pamplona

E-mail: jvelis@unav.es 


\section{INTRODUCCIÓN}

La variante plasmocitoide del carcinoma de urotelio es un hallazgo anatomopatológico de extremada rareza. Se trata de una variante muy agresiva, con tendencia a la recurrencia y a la metastatización temprana que puede confundirse histológicamente con neoplasias de estirpe hemolinfoide ${ }^{1}$. En nuestro conocimiento tan sólo se han descrito casos aislados y series cortas de esta variante neoplásica. Aportamos nuestra experiencia de un caso y revisamos la literatura.

\section{CASO CLÍNICO}

Varón de 60 años de edad que acude a consulta en enero de 2009 por episodios de hematuria macroscópica ocasional de dos años de evolución. Transcurridos 10 meses desde el primer episodio empezó con polaquiuria y disuria, por lo que 2 meses después se realizó estudio ecográfico en el que se evidenciaba un engrosamiento de la pared vesical derecha que condicionaba ureterohidronefrosis. Se realizó estudio de extensión mediante TAC toraco-abdominal y gammagrafía ósea no apreciándose lesiones sugestivas de metástasis y confirmando los hallazgos ecográficos. En febrero de 2009, recibió tratamiento quirúrgico mediante RTU vesical con diagnóstico de carcinoma plasmocitoide de vejiga con invasión de la capa muscular propia y presencia de focos de embolización linfovascular, por lo que se le indicó una cisto-prostatectomía radical con derivación ileal que se practicó sin incidencias el 27 de marzo de 2009.

El informe anatomopatológico reveló una neoformación epitelial atípica de crecimiento difuso con algunos nidos o cordones, constituidos por células de pequeño tamaño, citoplasma escaso y núcleo grande, pleomórfico y polilobulado. Además se observaron algunas áreas donde los núcleos eran más pleomórficos, polilobulados, grandes y muy hipercromáticos, respecto al resto de la población tumoral. Las figuras de mitosis tanto típicas y atípicas así como de apoptosis eran frecuentes (Fig. 1). Las células neoplásicas infiltraban la pared vesical alcanzando la capa muscular propia. El estudio inmunohistoquímico citoplasmático fue positivo en un $100 \%$ para la citoqueratina- 7 y en un $70 \%$ para la citoqueratina-20 y la citoquetatina de alto peso molecular con carácter difuso (Fig. 2). El diagnóstico definitivo fue de carcinoma de urotelio poco diferenciado, de alto grado, variante de células plasmocitoides.

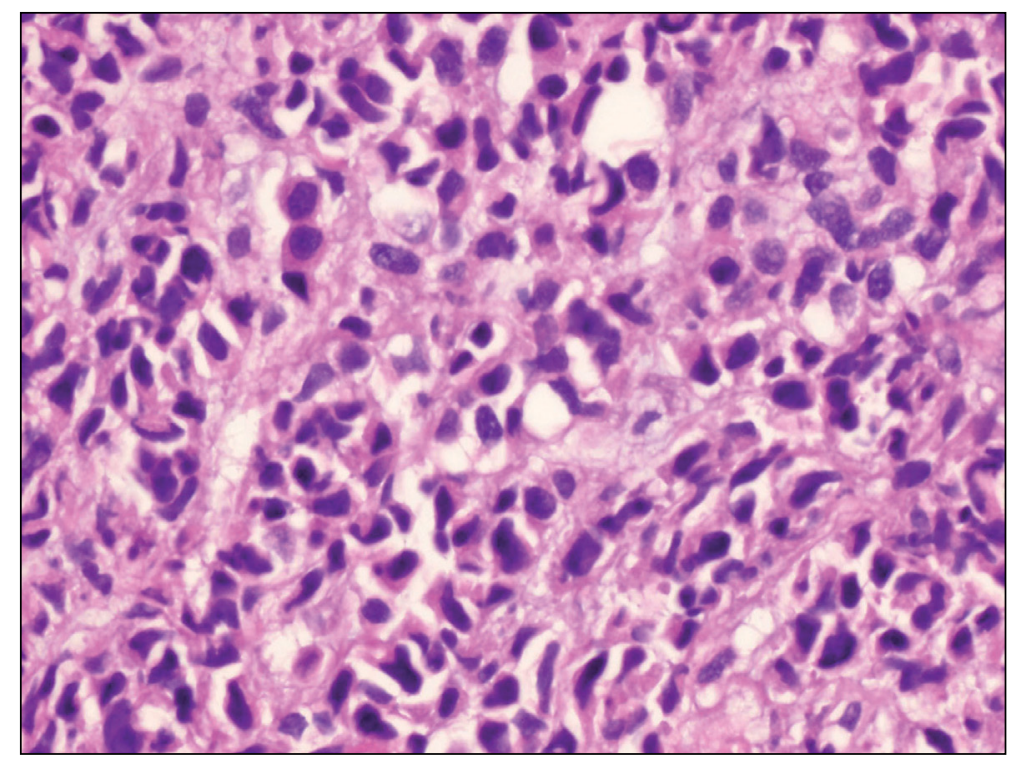

Figura 1. Imagen microscópica, donde se aprecia una neoformación epitelial atípica de crecimiento difuso con algunos nidos o cordones, constituidos por células de pequeño tamaño, citoplasma escaso y núcleo grande, pleomórfico y polilobulado ( $\mathrm{H}-\mathrm{E}$ x 40$)$. 

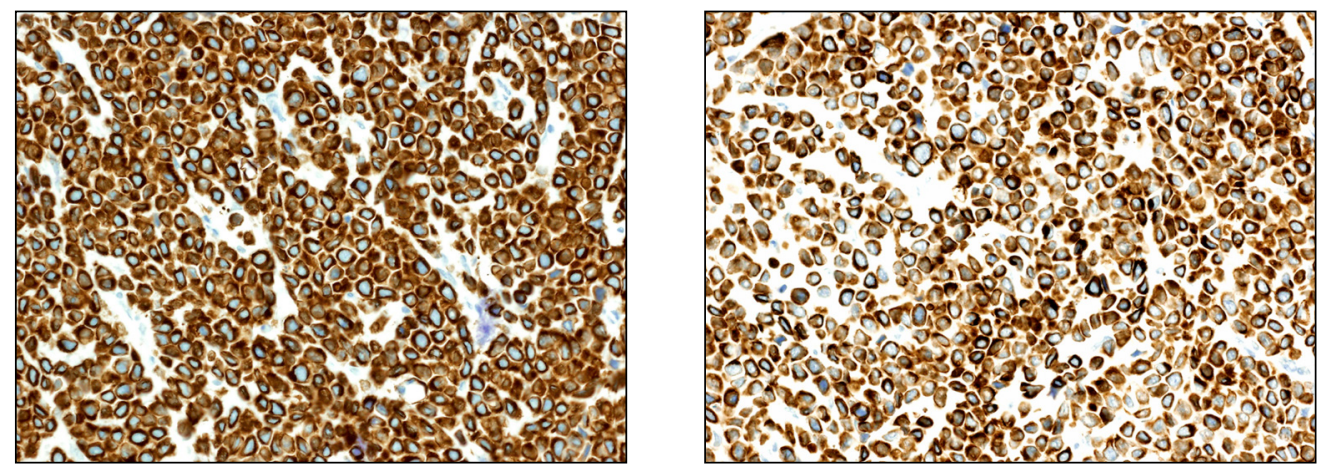

Figura 2. a) Inmunorreactividad citoplasmática + frente a la citoqueratina-7. b) Inmunorreactividad citoplasmática + frente a la citoqueratina- 20 .

A continuación, el paciente fue remitido al departamento de Oncología iniciando tratamiento quimioterápico adyuvante con Cisplatino y Gemcitabina ( 4 ciclos) que concluyó en agosto de 2009

En marzo de 2010, en exploración de control y estando el paciente asintomático, se objetivaron lesiones sugestivas de metástasis en columna, por lo que se realizó RM de columna en la que se informó de posibles metástasis a nivel de D3, D8, D9, D10 y L1. Se realizó gammagrafía ósea y PET-TAC que no fueron concluyentes, por lo que se recomiendó control evolutivo en tres meses. En dicho control se objetivó crecimiento de las lesiones óseas ya conocidas que se biopsiaron, no siendo concluyentes de malignidad.

En septiembre de 2010 se repitió el PET-TAC no objetivándose signos de captación ósea patológica, pero sí ganglionar múltiple en región axilar izquierda, perivascular mediastínica, subcarinal y mamaria interna, así como a nivel retroperitoneal. Se realizó de nuevo punción con aguja fina vertebral que se informó como lesión metastásica compatible con tumor urotelial plasmocitoide. Además se realizó biopsia de ganglios mamarios que también se informaron como compatibles con lesiones metástasicas originarias del tumor urotelial plasmocitoide.

En ese momento se inició una nueva línea de tratamiento quimioterápico con Paclitaxel y Carboplatino, en pauta trisemanal con un total de 6 ciclos, que concluyó en enero de 2011. A mitad de tratamiento quimioterápico se realizó nuevo TAC de control en el que la enfermedad permanecía estabilizada.

El 17 de febrero de 2011, en TAC de reevaluación se objetivó ureterohidronefrosis moderadasevera derecha a expensas de imagen nodular que protruía en la luz ureteral sugestiva de nue- va lesión tumoral. Además presentaba atrofia funcional del riñón contralateral con aumento de la creatinina plasmática hasta $2,9 \mathrm{ng} / \mathrm{mL}$. Se decidió la colocación de una nefrostomía percutánea derecha con colocación de un catéter ureteral doble $\mathrm{J}$ por vía anterógrada. Durante este ingreso sufrió un IAM inferoposterior y de ventrículo derecho tratado mediante angioplastia primaria de manera eficaz.

Finalmente, a mediados de marzo de 2011 falleció el paciente.

\section{DISCUSIÓN}

El carcinoma urotelial en su variante plasmocitoide es una entidad rara, habiéndose descrito hasta la fecha, casos aislados y series cortas de esta variante neoplásica. Las primeras descripciones de esta variante histológica fueron halladas en $1991^{1}$.

Su presentación clínica es con frecuencia tardía, así como la aparición de la hematuria acompañante, siguiendo la enfermedad un curso ominoso y recurrente que desemboca en la muerte del paciente ${ }^{2}$. Sin embargo, debido al escaso número de casos descritos, su comportamiento biológico, pronóstico y manejo terapéutico son difíciles de establecer ${ }^{3}$.

En el estudio con la serie más numerosa hasta la fecha, Keck y col presentan 32 casos, obtenidos de 17 centros diferentes, mostrando que el carcinoma urotelial plasmocitoide es de alto grado en el $100 \%$ de los casos y presenta metástasis a distancia en el $60 \%$ de ellos en el momento de su 
diagnóstico ${ }^{4}$ y aparecido de manera diseminada y extensa a lo largo de las paredes de la vejiga así como en los órganos pélvicos adyacentes ${ }^{2}$.

Es de particular interés el aspecto plasmocitoide de las metástasis, ya que pueden llevar al diagnóstico erróneo de mieloma múltiple o de linfoma maligno, los cuales pueden presentarse en la vejiga de manera secundaria, aunque raramente lo hacen como tumores primarios ${ }^{3}$.

En el diagnóstico diferencial habría que contemplar entidades benignas tales como la cistitis crónica con un infiltrado prominente de células plamáticas, así como tumores malignos como el plasmocitoma, adenocarcinoma de células en anillo de sello y metástasis de otros tumores primarios, específicamente de mama y de estómago $^{5}$. Al igual que éstos, presenta un déficit de expresión de cadherina $\mathrm{E}$ por parte de sus células, lo cual se sugiere en algunos estudios como el desencadenante de la elevada agresividad que presentan ${ }^{6}$.

La presentación de un plasmocitoma primario de vejiga es rara, pero en pacientes con una historia previa de mieloma múltiple, en los que no se identifica componente urotelial superficial, es necesario mantener cierto nivel de sospecha para no realizar diagnósticos erróneos entre plasmocitoma y un carcinoma urotelial con caraterísticas plasmocitoides ${ }^{5}$.

Se trata de un tumor con células de abundante eosinofilia citoplasmática, núcleo pequeño hipercromático y frecuentes figuras de mitosis ${ }^{7}$. Sus células presentan una morfología similar al plasmocitoma, siendo esencial la distinción entre ambos para el manejo clínico y pronóstico, puesto que la cistectomía sería un tratamiento beneficioso para el carcinoma urotelial plasmocitoide, mientras que cuando se trata de un plasmocitoma en el contexto de un mieloma múltiple el tratamiento necesario sería la quimioterapia sistémica ${ }^{3}$.

El análisis inmunohistoquímico es necesario para caracterizar el carcinoma urotelial plasmocitoide, ya que las células tumorales presentan inmunorreactividad citoplasmática positiva frente a citoqueratinas, especialmente para las citoquerati- nas de alto peso molecular, la citoqueratina $\mathrm{CK} 7 \mathrm{y}$ la CK20 ${ }^{8}$. Y negativa frente a vimentina -marcadores del linfoma-, CD138 -marcadores neuroendocrinos-, proteína S-100, desmina y actina del músculo liso ${ }^{7}$

Tres son las características que dan consistencia al diagnóstico del tumor urotelial plasmocitoide: la presencia de carcinoma urotelial "in situ"; la presencia de áreas de crecimiento típico de carcinoma urotelial y la inmunorreactividad positiva frente a citoqueratinas ${ }^{3}$

El enfoque terapéutico en pacientes con carcinoma urotelial plasmocitoide sigue siendo discutible debido a la rareza del tumor. La enfermedad diseminada a distancia tiene un mal pronóstico y no se conoce una línea terapéutica que se haya mostrado efectiva. La cistectomía radical supone la primera opción de tratamiento para el carcinoma urotelial músculo-invasivo no metastásico, incluyendo la variante plasmocitoide. Se sugiere también un posible beneficio con quimioterapia adyuvante $^{7}$ Kohno y col describieron un caso con respuesta completa mediante quimioterapia neoadyuvante con 2 ciclos de metotrexate, etopóxido, vinblastina y cisplatino $^{9}$

\section{BIBLIOGRAFÍA}

1. Gaafar A, Garmendia M, de Miguel E, Velasco V, Ugalde A, Bilbao FJ et al. Plasmacytoid urothelial carcinoma of the urinary bladder. A study of 7 cases. Actas Urol Esp 2008; 32: 806-810.

2. MaI KT, Park PC, Yazdi HM, Saltel E, ERdogan S, STInson WA et al. Plasmacytoid urothelial carcinoma of the urinary bladder report of seven new cases. Eur Urol 2006; 50: 1111-1114.

3. Ro JY, Shen SS, LeE HI, Hong EK, LeE YH, CHO $\mathrm{NH}$ et al. Plasmacytoid transitional cell carcinoma of urinary bladder: a clinicopathologic study of 9 cases. Am J Surg Pathol 2008; 32: 752-757.

4. Keck B, Stoehr R, Wach S, Rogler A, Hofstaedter F, Lehmann J et al. The plasmacytoid carcinoma of the bladder-rare variant of agressive urotelial carcinoma. Int J Cancer 2011; 129: 346-354.

5. López-Beltrán A, Requena MJ, Montironi R, BlanCA A, Cheng L. Plasmacytoid urothelial carci- 
noma of the bladder. Hum Pathol 2009; 40: 1023-1028.

6. Ricardo-González RR, Nguyen M, Gokdend N, SAngor AR, Presti JC JR, McKenneya JK. Plasmacytoid carcinoma of the bladder: a urothelial carcinoma variant with a predilection for intraperitoneal spread. J Urol 2012; 187: 852855.

7. Fritsche HM, Burger M, Denzinger S, Legal W, Goebell PJ, Hartmann A. Plasmacytoid urothelial carcinoma of the bladder: histological and clinical features of 5 cases. J Urol 2008; 180: 1923-1927.

8. Sato K, Ueda Y, Kawamura K, Aihara K, Katsuda S. Plasmacytoid urothelial carcinoma of the urinary bladder: a case report and immunohistochemical study. Pathol Res Pract 2009; 205: 189-194.

9. Kohno T, Kitamura M, Akal H, TaKaha M, KawaHARA K, OKA T. Plasmacytoid urothelial carcinoma of the bladder. Int J Urol 2006; 13: 485-486. 